\title{
Characterizing Research Process Sophistication in Engineering Ph.D. Stu- dents and the Influence of Prior Experiences
}

\section{Ms. Erika Mosyjowski, University of Michigan}

Erika Mosyjowski is a PhD student in the Center for the Study of Higher and Postsecondary Education at the University of Michigan. She also earned a Master's in Higher Education at Michigan and a Bachelor's in Psychology and Sociology from Case Western Reserve University. Before pursuing a PhD, Erika had a dual appointment in UM's College of Engineering working in student affairs and as a research associate. While grounded in the field of higher education, her research interests include engineering education, particularly as related to innovation, professional identity development, and supporting the recruitment and persistence of underrepresented students within engineering.

\section{Dr. Shanna R. Daly, University of Michigan}

Shanna Daly is an Assistant Professor of Mechanical Engineering at the University of Michigan. She has a B.E. in Chemical Engineering from the University of Dayton (2003) and a Ph.D. in Engineering Education from Purdue University (2008). Her research focuses on strategies for design innovations through divergent and convergent thinking as well as through deep needs and community assessments using design ethnography, and translating those strategies to design tools and education. She teaches design and entrepreneurship courses at the undergraduate and graduate levels, focusing on front-end design processes.

\section{Dr. Diane L. Peters, Kettering University}

Dr. Peters is an Assistant Professor of Mechanical Engineering at Kettering University.

\section{Dr. Steven J. Skerlos, University of Michigan}

Professor Steven J. Skerlos is Arthur F. Thurnau Professor at the University of Michigan. He is a tenured faculty member in Mechanical Engineering and Civil and Environmental Engineering. He also serves as a UM Distinguished Faculty Fellow in Sustainability.

He is Director of Sustainability Education Programs in the College of Engineering and Co-Director of the Engineering Sustainable Systems Program. He is Chief Science Officer of Fusion Coolant Systems.

Professor Skerlos has gained national recognition and press for his research and teaching in the fields of technology policy and sustainable design. He has co-founded two successful start-up companies (Accuri Cytometers and Fusion Coolant Systems), co-founded BLUElab, served as Director of the Graduate Program in Mechanical Engineering (2009-2012), and served as associate and guest editor for four different academic journals.

His Ph.D. students in the Environmental and Sustainable Technologies Laboratory have addressed sustainability challenges in the fields of systems design, technology selection, manufacturing, and water.

Ms. Megan Kaczanowski, University of Michigan 


\section{Characterizing Research Process Sophistication in Engineering PhD Students and the Influence of Prior Experiences}

\section{Introduction}

One goal of engineering $\mathrm{PhD}$ programs is to support students' transition to independent researchers and it is expected that by the time they have completed a doctoral degree, students will have developed the skills necessary to design and manage an independent research project. Engineering PhD students need to be able to consider the broader context and potential impacts of their research, identify and frame an appropriate research question, develop an approach to studying their topic of interest, and use experience and intuition to steer their projects and navigate challenges that may arise. One might characterize such skills as elements of research process sophistication. However, while experienced researchers may be able to identify a sophisticated application of such skills in which they "know it when they see it," a need exists for a way to consistently and systematically represent students' varying levels of research process sophistication. This would allow programs to evaluate groups of students at varying levels in their degree process as well as single students over time to evaluate progress.

The need for a classification system to characterize the sophistication of graduate research in engineering became evident to our team in a study of engineering $\mathrm{PhD}$ students with extensive work experience prior to enrolling in doctoral study, a group we call returners. Though graduation age data suggests returners are in the minority of engineering doctoral students, they are an important group to understand. In addition to representing an under-researched pathway through advanced engineering training, given the combination of returners' rich real-world work experience with advanced engineering training, these students may be uniquely poised to develop innovative solutions to important problems of contemporary society. Creative cognition theory suggests in part that innovation is likely to occur at the intersection of multiple experiences or perspectives. ${ }^{1}$ Based on such work, we suspected returning students' past experiences might contribute to returners having different, possibly more sophisticated, independent research processes.

While the research process sophistication classification scheme discussed in this article was developed in context of needing to be able to evaluate potential differences in the ways returning and direct pathway engineering students approach research, ways to better characterize variations in the levels of students' research work is applicable to a broader population of engineering graduate students. In this paper, we detail our process for developing a categorization system for research process sophistication. We identified three emergent categories of research process sophistication based on interviews of over 50 students' with diverse prior experiences. The three categories of research process sophistication described in this paper will later be used to characterize the responses of all returning and direct pathway students in our study and better 
understand how students' past education, work, and other experiences relate to their engineering research process. However, the applicability of such a classification scheme would likely extend to evaluating the work of engineering graduate students' research sophistication or progress over time more broadly.

\section{Background}

Research Skills. A major component of doctoral education is preparing students to be independent researchers. There are a variety of research skills essential for career success in academia, government, and industry that students need to have upon completion of the PhD. Identifying and assessing necessary engineering skills (including various research-related competencies) has been a popular topic of study at the undergraduate level, but has been explored in less depth at the doctoral level. Several studies of engineering graduate education consider the skills of doctoral graduates as they relate to the needs of prospective employers. ${ }^{2,3}$ Many colleges of engineering also identify a number of research competencies as part of broader lists of graduate competencies that they aim for their doctoral students to develop over the course of their degree. One article by Rogers and Goktas ${ }^{4}$ examined students' research proficiency and the factors that affect this proficiency, measuring research proficiency based on students' assessment of their preparation and growth for a number of individual research skills. However, we were not able to find any works outlining a holistic assessment engineering doctoral students' research skills.

While there is little literature that describes a holistic assessment of engineering doctoral students' research work, several articles do identify specific skills associated with successful doctoral-level research. In their article exploring engineering graduate students' research proficiency, Rogers and Goktas ${ }^{4}$ named a variety of research skills, including: statistical ability, written and oral communication, critically analyzing information, analyzing and interpreting data, conducting experiments, and locating and learning from literature. A study by Berdanier et al. examined the knowledge, skills, and attributes graduate students intending to pursue work in academia and industry identified as important. ${ }^{5}$ Both groups of students emphasized problem solving, meeting deadlines, communicating with a particular audience (both orally and in writing), strong analytical ability, managing multiple projects, working in teams, and meeting deadlines. Skills and knowledge emphasized exclusively by students intending to pursue industry careers tended to industry-specific, like writing technical reports and learning new technologies. Those graduate engineers entering academia also emphasized skills that related to their future roles as advisors and researchers. Research skills emphasized by these students included those related to bringing in and managing research funding and managing a research team.

Various graduate departments in engineering outline research competency expectations for their graduates that compliment and expand on those elements of graduate-level research identified in 
the literature. The Mechanical Engineering department at the University of Michigan cites a number of skills associated with conducting $\mathrm{PhD}$ research, including:

...Combining knowledge gained in the classroom with the ability to read the scientific literature, identify critical knowledge gaps, structure complex problems, formulate and test hypotheses, analyze and interpret data, and present and discuss technical results. Engineering research also requires significant experimental, computational, and analytical skills. ${ }^{6}$

The department explains that many of these skills need to be developed outside of the classroom and that independent research is a critical component of earning a $\mathrm{PhD}$. Cornell's Civil and Environmental Engineering department similarly lists important proficiencies considered in assessing PhD candidates. Key proficiencies include the ability to do the following:

- Think originally and independently to develop concepts and methodologies

- Identify new research opportunities within one's field

- Synthesize existing knowledge, identifying and accessing appropriate resources and other sources of relevant information and critically analyzing and evaluating one's own findings and those of others

- Master application of existing research methodologies, techniques, and technical skills

- Communicate in a style appropriate to the discipline ${ }^{7}$

The University of Virginia cites the importance of many similar skills for their graduate students across disciplines and to these lists adds the ability to deal with ambiguity, to reach and defend conclusions, ethics and integrity, leadership, seeing a project through from inception to completion, being self-motivated, and demonstrating strategic thinking. ${ }^{8}$

The Role of Experience in Research Skill Development. Returning students are likely to have prior engineering experiences through which they have developed skills and knowledge relevant to their doctoral research work in engineering. Much of the literature that seeks to explain differences in research skills or skills necessary for completing other complex tasks cites the role of experience as a key influence on individuals' level of aptitude in a particular domain. Rogers and Goktas' work showed an annual increase in graduate engineering students' research preparedness after their first year of graduate study, which the authors in part attribute to increased emphasis on and opportunities for research (as opposed to coursework) after this first year. ${ }^{4}$ In Dreyfus and Dreyfus' five-stage model of adult skill acquisition, the authors emphasize the critical role of "concrete experience" in achieving mastery in a particular domain."

In engineering, being able to come up with innovative solutions to important societal problems is a key element of being a successful researcher. Creative cognition theory suggests in part that one potential source of innovation is the combination of ideas from multiple domains or perspectives. ${ }^{1}$ Those engineering graduate students who are returners are not only likely to possess more experience in the field of engineering that can lend itself to expertise, but, given 
their unique combination of applied engineering experience and advanced engineering training, returners may be particularly well-positioned to do innovative work with real-world implications. Our team's earlier work suggests that engineering returners draw on their rich prior work experiences in approaching their doctoral research. ${ }^{10}$ Other literature on returning students in a variety of fields suggests that returners are also typically highly-motivated and goal-oriented, with a high work ethic and strong interpersonal skills. ${ }^{11,12,13}$ Drawing on creative cognition theory, the existing literature, and our team's earlier work, we suspected that returners' past experience contributes to them demonstrating more sophisticated approaches to research than their direct pathway doctoral student peers. This paper describes our first step in addressing this question: the development of an emergent, holistic, classification scheme for students' levels of research process sophistication.

\section{Research Methods}

Study Goals. The goal of this study was to develop a way to characterize research sophistication of engineering $\mathrm{PhD}$ students. The following research questions guided our study:

1. How do engineering $\mathrm{PhD}$ students' conceptions of and approaches to engineering research differ?

2. How do students' prior experiences inform the ways they think about and approach their $\mathrm{PhD}$ research?

Through the development of a categorization scheme for research process sophistication and a description of the emergent categories of sophistication, we explore the characteristics that distinguish differences in students' research understanding and abilities and the role of experience in shaping how students approach a research problem.

Participants. Participants were recruited for interviews from a survey we distributed as part of a larger project on graduate student experiences and motivations. ${ }^{14}$ In total, we interviewed 53 participants, and 52 of those students responded to our questions about a hypothetical research scenario, which are the questions we focused on for the goals of this study on research sophistication. We sought to interview a diverse pool of engineering doctoral students, both in terms of their returner status but also in relation to institution, major, gender, and racial/ethnic background. Though we intentionally sought multiple participants from some institutions, we sought additional institutional variety in our Skype interviews and in total interviewed students from 19 different universities. Approximately 40 percent $(n=21)$ of the participants we interviewed were female, over 15 percent $(n=8)$ were underrepresented minorities, and approximately half were returning students $(n=27)$ and half direct pathway students $(n=26)$. Students were pursuing PhDs in a variety of engineering fields including mechanical, electrical, civil, bioengineering, aerospace, industrial, materials, computer science, and systems engineering as well as engineering education and several combined or multidisciplinary programs. 
Interview Protocol Development. Questions about participants' research processes were a part of a larger interview protocol that focused on understanding students' skills, experiences, and perspectives related to $\mathrm{PhD}$ research and the influence of their past experiences on their current research work. Development and execution of the full protocol as well as the questions related to participants' approaches to research were guided by literature on best practices for interview design and administration. ${ }^{15,16,17}$ Here we focus on the development of the questions related to research sophistication.

While the broader focus of our project is to understand more about the experiences of returners and the ways their work experiences shape their PhD work, we wanted to ensure that our interview allowed us to elicit information about students' research processes in a way that allowed us to capture the influence of past experience without unduly privileging this past experience as necessarily indicative of greater research aptitude or sophistication. Further, we wanted to allow for an understanding of the ways a variety of past experiences, like short-term jobs, internships, co-ops, or undergraduate or master's research experiences, shaped participants' research work, so as not to assume only long-term work experiences have an impact on one's thinking about research.

Our questions on research process sophistication, including the potential influence of their past experiences, were based on a broadly-defined, hypothetical research scenario, in which we asked participants a series of questions about their responses in such a situation. The scenario, as presented in our protocol stated "Imagine you have completed your PhD and are an independent researcher, and have the opportunity to receive funding for a five-year project. For these next several questions I'd like you to answer according to this scenario." We asked students seven questions related to this scenario:

1. How would you determine what is an important problem?

2. How would you figure out what needs to be done and what can be done?

3. Imagine you've selected a problem to work on, how would you figure out possible approaches and methods to studying it? Once you've explored possible approaches and methods, how would you select one of those to move forward with?

4. How confident would you feel in your intuition when it came to steering the project?

5. To what extent would you feel comfortable taking risks in your proposed project? What factors influence your willingness to take or not take risks?

6. How would you respond to unexpected challenges?

7. How would you respond if you got negative feedback on your ideas?

After each response to the hypothetical scenario, we asked students to describe any specific activities or experiences they had, both during and prior to their $\mathrm{PhD}$, in which they completed a similar task or drew on a similar skill as the one described. It was our goal to learn generally about how participants thought about their research processes through the questions related to the 
hypothetical scenario and to then learn more about connections between their past experiences and current thinking through follow-up questions on related work and graduate study experiences.

After developing the initial draft of our interview protocol as a team, we piloted the interview protocol with several participants who were not in our larger study, but who were either in the process of completing, or who had recently completed, a $\mathrm{PhD}$ in engineering and who had at least some work experience prior to their $\mathrm{PhD}$. These pilot interviews helped us to refine the instrument and get a sense of the time it would take to complete one interview.

Data Collection. We conducted 39 of 53 of our interviews in-person at institutions with a high number of study participants and later, to ensure students from a variety of institutions were included in this phase of our study, recruited an additional 14 participants at other institutions to be interviewed via Skype. The interviews were conducted over a 6 month period by one member of our research team. Interviews ranged from approximately 35 minutes to 2 hours in length.

Data Analysis. To explore variation in students' research process sophistication, we focused on students' responses to the seven questions related to the hypothetical research scenario (including follow up questions regarding their experiences). We separated these questions from the rest of the interview, as it was likely to contain information that could easily identify students as returners or direct pathway students. We created an individual table of responses for each participant and read through all of these responses as a group, to get an initial sense of the types of responses. Then, one researcher read through each participant's set of responses, inductively sorting them into piles based on perceived differences. Reasons for grouping certain responses together as well as similarities and differences among the multiple piles were described by key words and phrases. This sort resulted in three groups of responses, representing three levels of research sophistication. After this initial sort, the researcher re-read each group as a set, to get a sense of how well the responses held together as a set and to identify any additional themes common between each group. While the initial three categories held together thematically, several sets of responses were better characterized as falling between two of the groups. Each category of responses were read as a group for a final time to ensure our descriptions of each category were complete and accurate.

\section{Findings: Three Categories of Research Process Sophistication}

The findings described in this section define and characterize the three categories (or levels) of research process sophistication, using one interview to exemplify each of the three categories. In this categorization scheme, Category 1 includes those responses that demonstrated the lowest levels of research process sophistication, while Category 3 includes the most sophisticated responses. These three categories represent a continuum, with variation between and within each 
of the categories. While future work will explore the role of time in $\mathrm{PhD}$ program in greater depth, to account for the way a students' time in a doctoral program may shape how they think about research, all of the illustrative examples for the three categories of research process sophistication come from students who were in at least their third year of doctoral study.

Category 1. Participants whose responses fell in Category 1 were most likely to struggle to answer questions related to the independent research scenario. Their responses were typically somewhat vague, with little detail about the specific elements they would consider in engaging in an independent research task. Answering the first question of how they would identify an important problem proved to be a particular challenge for students in this category. Students in this category expressed a lot of doubt in their responses and uncertainty about the "right" answer to these questions. When asked about their likely responses to negative feedback on their research, participants in this category were most likely to express difficulty with or avoidance of receiving critiques of their research work.

Responses in Category 1 also frequently suggested a reliance on external guidance and expertise. Students referred to what they were taught in their coursework or an advisor's rules or suggested best practices for research. Category 1 responses and associated examples often highlighted the importance of others' input on guiding a project. Students explained they would consult with expert researchers to "check" their work or look to a more experienced individual for support.

Responses that fell in Category 1 represented a more concrete, black-and-white understanding of how to best address an engineering research project. One student, when asked how he might determine what needs to be done and what can be done, responded that there was "probably an equation" to address this issue. Participants in this category often provided relatively simple answers for the best ways to explore a particular research area and guide a study. Their responses did not explore more nuanced, complex issues of steering a research project.

The responses of participants in Category 1 often belied limited prior experience. Participants in this category often self-identified as inexperienced, as students who were still learning. Additionally, when prompted for examples of relevant past experiences with various elements of the research process, participants at times struggled to cite examples of instances in which they had experienced or engaged in these particular practices. When students were able to provide examples of past experiences, they often lacked in detail.

Example of a Category 1 response, Olivia: The responses of Olivia serve to illustrate Category 1. Olivia expressed difficulty in answering questions about how she would manage an independent research project, stating "Oh my gosh. This is hard. These are tough. Hopefully, everyone struggles with them." Like other students in Category 1, Olivia was uncertain about how she might select a research topic. She noted that some of her peers selected a topic based on 
a family connection to a particular problem but, lacking a relevant family connection to an issue, she was uncertain about what she might select, explaining:

I don't know. I mean, I guess I would see if there were any issues that I had or family members or someone close to me had that I could do research on and see. I mean, I think that's why a lot of people ...Yeah. I mean, with my background, I don't know what I would study.

When asked about her response to negative feedback, Olivia did not express particular discomfort like many students in Category 1, but she also did not provide a direct response to how she would handle it. Rather, she cited several instances in which she received negative feedback, but said little about her reaction or how she might react to a similar situation in the future.

Olivia frequently mentioned instances in which she looked to the advice, work, and guidance of others. When asked about her ability to steer an independent research project, she explained that while she thought she could steer it, she would look to a more experienced individual for feedback on her approach.

I think I could steer it. I wouldn't want to go without talking to people in my field who know better than I do like this is what I was thinking, do you have any suggestions, do you have a better alternative than this way? So someone who I knew who knew that area just a little bit better and I mean, that's why we do collaborative research, that's why I have two advisers.

Similarly, Olivia expressed a desire to consult with experts in the field before deciding on the level of risk she would be willing to take on a particular project. She explained that in her past experiences, she had primarily been instructed to do a particular task by an advisor, which may relate to her expressed desire to continue to seek expert guidance. At several points, Olivia also described the work of other students to illustrate a point. In describing how she would identify what needs to be and can be done related to a particular problem, Olivia referred to "what everyone would say to do" (a literature search, in this case), which highlights again an emphasis on what others might do in a given situation, but is also a rather simple answer that seems to suggest a straightforward, "correct" way to address a problem.

Category 2. Defining the middle of a continuum is perhaps the most challenging and allows for the most variation. The responses in Category 2 reflected a range of themes and emphases. Overall, participants whose responses fell into Category 2 demonstrated a greater awareness of elements that might be considered key to embarking in engineering research and were typically able to respond to all questions regarding different elements of the independent research project scenario. However, while as a whole, students in Category 2 were more confident than those in Category 1 , some students in this category did still express doubt about their ability to conduct 
independent research. Responses of participants in Category 2 suggested that these students were learning more about the field of engineering and the associated norms, practices, and priorities.

Responses from students in Category 2 either described their approaches as replicating what they did before without indicating adjustments to that particular scenario or were fairly general. Participants in this category often struggled to apply knowledge or skill developed in a particular situation to how they might address a similar problem in the future. Often, the responses to questions related to how students would guide a hypothetical research project drew upon seemingly concrete "rules" or things to consider in approaching engineering research, but did not necessarily reflect a consideration of the research context or, in some cases, the student's own agenda, work style, or personal strengths. In some cases, students questioned their agency in being able to select a topic to research and lead that project, citing the influence of funding agencies and industry trends, as well as their own future positions. Participants in Category 2 often stated a desire to hear multiple perspectives and work out a solution, but did not necessarily take ownership over a particular perspective, or approach or explain criteria they might use to assess these multiple perspectives and feedback on their research.

Many participants in Category 2 provided detailed examples of their past experiences with multiple elements of the research process. However, while some participants in this category may have provided rich examples, no participants in this category provided both rich past examples and a detailed description of how they would approach various elements of the hypothetical research scenario, suggesting perhaps they struggled to apply their past experiences to new situations. Some individuals in Category 2 did reflect on the limitations of their engineering research experiences to date in discussing the difficulty of designing and steering an independent research project.

Example of a Category 2 Response, Yvette: Given the variety of responses that can fall between Categories 1 and 3, there is no single "typical" Category 2 response. Yvette's responses, however, are a good example of a student who is developing her skills and knowledge related to conducting independent research and, while is perhaps not yet demonstrating the highest level of research process sophistication, is more able to explain her approach to various elements of the research process. When asked about identifying an important problem, Yvette pointed to an area related to her expertise that she personally considered important and expressed an intention to balance that with the opportunity to have a new experience or explore something she might not otherwise be able to do. She also briefly discussed her experiences thinking about important problems to address in her past work and graduate experience, though these examples were relatively general rather than referring to a particular instance.

Though it was clear that Yvette had a degree of comfort and familiarity with research in engineering, she did indicate she was continuing to develop her knowledge and her toolkit for 
addressing engineering problems. She described that in her undergraduate experience she observed different mentors to learn new ways of approaching a problem and said this is a practice that she has continued into her $\mathrm{PhD}$ with her advisor, explaining "I very much consider a lot of our relationship is him training me how to do things well." In a hypothetical scenario, Yvette explained that there were many "standard toolkits" in her field that she has and will likely to continue to utilize in her work with the goal of using these toolkits in new ways.

Yvette described her outlook on both risk taking and unexpected challenges that arise over the course of a project as evolving, with increasing comfort with both. She explained that while she viewed her field as pretty high risk, toward which she sometimes felt uncertainty, she was trying to take more risk, particularly within an academic setting, explaining "we're not in a high stakes environment with death, or we're not going to lose a whole bunch of money" if an experiment were to fail. Similarly, she expressed a growing appreciation of unexpected challenges, explaining she used to view "challenge as something much more negative" in her past work experience but, during grad school, has come to see them as "more of an opportunity." Based on new experiences in graduate school, her perspectives and comfort with potentially difficult situations were evolving.

Category 3. Category 3 responses were characterized by clarity, detail, strategic thinking, and consideration of potential contextual influences on a research project. Participants in this category provided detailed responses that covered multiple elements of the process of designing and steering and independent research project. This consideration included an emphasis on the importance of long-term planning to account for the influence of funding agencies, stakeholders, and needs and resources associated with team management and the responses broadly reflected an understanding of the complexity of independent engineering research. Responses in Category 3 suggested knowledge about the needs and practices of participants' specific area of engineering. These students demonstrated comfort with the specifics of conducting independent research while discussing the hypothetical research scenario in a way that suggested they were able to abstract to explain their research process more broadly demonstrating their ability to apply core principles or knowledge across multiple settings.

As a whole, participants in Category 3 expressed not only greater confidence in their own ability to lead an independent engineering research project, but also a sense of ownership of a particular approach or area of work. Students whose responses fell in this category expressed a strong sense of purpose and had a clear path or area of focus when asked to consider how they would identify an important research problem, clarity that was also evident in the way they discussed their research process. Some participants in Category 3 responded to the questions beginning with "What I always do is..." referencing a personally-held approach to guiding a research project, as opposed to an approach that is largely dependent on the guidance of others. However, taking ownership over one's research does not necessarily mean ignoring the perspectives and expertise 
of others. While participants in Category 3 expressed confidence, direction, and security in their own topic and process for research, they expressed an openness to the perspectives of others, even negative feedback from others, to inform their thinking. A distinguishing factor of participants in Category 3 related to their use of feedback from others was that they did not give all external opinions equal weight, but rather considered and evaluated all external input for its merit.

Students in Category 3 provided detailed examples of rich experiences in their past work and doctoral programs related to multiple elements of the research process. Category 3 participants often spoke of past experience conducting engineering research, which seemed connected to their relative comfort with and knowledge about the various elements of managing an independent research project. Their responses indicating an ownership over their personal approaches to research also suggested past experiences in which they were able to develop a "typical" approach. Some students in this category explicitly cited their past research experiences as influential in informing and shaping their current research process

Example of a Category 3 Response, George: George's responses to the questions about the independent research scenario illustrate a Category 3 response. George had rich, reflective responses relating to every aspect of the research scenario and, in addition to being able to speak at length about the various elements he would consider in any hypothetical situation, provided detailed examples from his past experiences in industry and his graduate study. In considering how he would identify an important problem, George considered the need he perceived at a national level, his own area of expertise, and the ways his own experiences shape the types of problems he would identify as important.

George's responses demonstrated that he felt a sense of ownership of and confidence in his approach to guiding an independent research project. He responded to the scenario by describing what he "tends to" do. When asked about his process for selecting a particular approach to studying a problem, George cited a personal heuristic that he developed to help himself think through all important elements of a potential solution, identifying six questions he always asks himself. He explained, "Those are things that engrained in me that I think of, and it comes out like you would like brushing your teeth every day. It's a habit. If I think of an idea, I analyze it in my head." Even when describing his interest in collaborating with others on a problem he frames it as a personal strategy that has "always worked for [him]," suggesting he considers working with others to be one tool to expand or challenge his own thinking, rather than a reliance on others to provide him with the "correct" course of action.

George expressed a comfort taking risks and explained the importance of being able to plan and manage risk, keeping back-up options in mind should an initial plan not work out. He expressed similar comfort with, even enthusiasm for, unexpected challenges that arise in the course of a 
research project, stating "I typically jump at the opportunity of solving a problem that nobody else is willing to take on. I love it." In describing his reaction to negative feedback, George explained that after the initial sting of receiving feedback, he would consider the feedback and, looking critically at his work determining if it warranted a "course correction" or if he feels sufficiently confident in his work to ignore the feedback.

\section{Discussion}

Variation occurred along multiple dimensions from Category 1 to Category 3. One distinguishing feature between categories was the extent to which participants considered complexity in their responses. Less sophisticated responses lacked detail, relied on convention, and presented a fairly straightforward process for addressing a research problem, while more sophisticated responses emphasized the need to consider a variety of environmental and stakeholder inputs and were likely to include a detailed description of how to respond to a number of specific elements of the research process. Participants who provided these more sophisticated responses seemed to draw on some past research experience and considered how similar issues might arise in a new research project. Other characteristics that varied between the categories were participants' confidence and the extent to which they expressed a feeling of ownership over their research topic and process. Students whose responses were characterized as less sophisticated often expressed a lack of confidence in their responses and spoke of relying on some external authority, whether it be something they perceived to be a convention of their field or consultation with an expert, for guidance in shaping their approach to research. Participants who gave more sophisticated responses spoke both more confidently and with more of a sense of ownership over their approach. They were more likely to describe their personally held academic interests, values, and approaches for studying a research problem.

While we generated categories to understand key differences in research sophistication for practical purposes, development from novice to an expert does not occur in concrete stages, and is theoretically best represented as a continuum. The boundaries between the different categories are neither permanent nor absolute. A student's response, when considered holistically, may be best classified into one category, with a particular element of that response better represented by another category. Further, while there is a value judgement associated with the different categories insofar as the development of research sophistication is a goal of engineering doctoral programs, all of these categories represent a valuable point within a student's development of his or her research abilities. For example, while a student in Category 1's reliance on authority and concrete rules might be less sophisticated than a more self-directed, self-assured approach evident in Category 3, learning from experts in the field and developing a familiarity with foundational knowledge and skills in engineering is a key step in being able to progress as an independent engineering researcher. Though students' responses at a particular point in time may 
be best represented by one category, it is possible and likely that students' level of research process sophistication will change over time as a result of their experiences.

Though our process for classifying graduate engineering students' research process sophistication was an emergent one, several theories may help us further interpret and explore our findings. Many of the themes that emerged from our analysis are reflected in the expertnovice literature. In Bransford, Brown, and Cocking's How People Learn, the authors describe a number of principles of experts' knowledge. ${ }^{18}$ These principles include expert's ability to readily retrieve important knowledge, a deep understanding of their subject matter, an understanding that knowledge cannot be reduced to a set of isolated facts but rather must be conditionalized to a particular set of circumstances, and vary in their level of flexibility in how they are able to apply familiar concepts in their approach new to new situations, which Bransford et al. refer to as “adaptive expertise." Dreyfus and Dreyfus' model of skill acquisition similarly emphasizes experts' consideration of context in their decision making and also describes experts' reliance on their intuition in determining the correct action in a particular situation. ${ }^{9,19}$ There appear to be some parallels how these works characterize experts versus novices and our emergent characteristics that distinguish different levels of research process sophistication. More sophisticated researchers in our study were more likely to readily provide detailed responses and past examples of related experiences, acknowledged the complexity of research, understood the role of context in shaping their decisions related to research, and expressed greater confidence in their ability to rely on their own intuition in directing their work. The principle of adaptive expertise is particularly helpful in considering differences between Category 2 and Category 3 responses. Many individuals in Category 2 provided detailed, relevant responses in how they had previously addressed a particular aspect of the research process, but struggled to abstract the processes described in a particular situation to how they might respond in a new hypothetical scenario.

In addition to parallels in our findings and the expert-novice literature, our findings are also informed by the concept of self-authorship. ${ }^{20}$ Baxter Magolda identified three key elements of self-authorship: trusting the internal voice (recognizing that while the world is outside of one's control, one's reaction is controllable), building an internal foundation (the creation of an internal foundation to guide one's reactions to reality), and securing internal commitments (understanding and practicing one's convictions). Baxter Magolda emphasized the applicability of this self-authorship framework in how individuals approach both work and daily life. One of the defining traits of self-authorship is the shift from a reliance on following external formulas to a reliance on and trust in one's internal voice in making decisions that allow one to consider and wisely respond to the environment. Elements of self-authorship were evident in variations in participants' research process sophistication. More sophisticated responses tended to emphasize one's internalized values and methods for thinking about and approaching a research scenario while less sophisticated responses were more likely to present a more deterministic view of how 
external influences may shape a project (in cases where context was considered at all) and a need for expert guidance. Participants who we classified as demonstrating higher levels of research process sophistication were also more likely to be more comfortable in responding to both negative feedback and unexpected challenges posed by external conditions and a greater confidence in their own intuition in directing their research.

Though it is not in the scope of this paper, our ultimate goal in developing this research process sophistication categorization scheme is to be able to examine what differences, if any, exist between returning and direct pathway students. We suspect that, given returners' rich past experiences in engineering and the role of experience in developing expertise, returners may enter their PhD programs already demonstrating higher levels of research process sophistication than their direct pathway peers. This foundation may also put returners in a good position to further develop their research skills by integrating their past experiences with their graduate training. In our future analyses, we will also need to be sensitive to students' time and progression in their doctoral program and the ways these may influence how they think about research.

While our immediate goal in developing a system to categorize students' research process sophistication is to explore potential differences between returning and direct pathway students, such a categorization system may have broader applicability within engineering graduate education. The literature points to a lack of research on defining and evaluating graduate research and more work in this area is needed. ${ }^{2,4,5}$ Currently, much of the work focuses students' self-assessment of individual skills, but there is little that discusses the holistic evaluation of students' research sophistication in a systematic, consistent way. At the doctoral level the successful completion of a dissertation is often the only assessment of students' development of research skills, but the evaluation of dissertation work is an often opaque process that likely varies significantly at the disciplinary, institutional, and individual level. Our research process sophistication categorization scheme may allow us to better track students' development as researchers throughout their doctoral programs.

\section{Conclusions}

We developed three categories to classify sophistication in research processes, with key distinguishing factors including participants' treatment of complexity, consideration of the broader context, ability to apply their knowledge and skill broadly, and self-authorship and feelings of ownership of their research work. Prior experience seemed to contribute to higher levels of sophistication. Our approach enabled us to take a more holistic look at students' research process sophistication, rather than evaluating individual traits or skills in isolation from one another. Findings from our research suggested that the expert-novice and self-authorship literatures may be helpful in future analyses of research process sophistication. In our future work, we plan to use our categorization scheme to explore potential differences in returning and 
direct pathway students' approaches to research, but it may be useful beyond the scope of our project. Further development of this classification systems may provide a way to track and support research growth in $\mathrm{PhD}$ programs.

1. Finke, R. A., Ward, T. B., Smith, S. M. (1996). Creative Cognition: Theory, Research, and Applications. Cambridge, MA: MIT Press.

2. Akay, A. (2008). A renaissance in engineering $\mathrm{PhD}$ education. European Journal of Engineering Education, 33(4), 403-413.

3. Watson, J., \& Lyons, J. (2011). Aligning Academic Preparation of Engineering Ph. D. Programs with the Needs of Industry. International Journal of Engineering Education, 27(6), 1394-1411.

4. Rogers, S. W., \& Goktas, R. K. (2010). Exploring engineering graduate student research proficiency with student surveys. Journal of Engineering Education, 99(3), 263.

5. Berdanier, C. G., \& Branch, S. E., \& London, J. S., \& Ahn, B., \& Cox, M. F. (2014), Survey Analysis of Engineering Graduate Students' Perceptions of the Skills Necessary for Career Success in Industry and Academia. Paper presented at 2014 ASEE Annual Conference, Indianapolis, Indiana.

6. Mechanical Engineering -- University of Michigan. (n.d.). Doctoral Degree. Retrieved January 25, 2016, from http://me.engin.umich.edu/academics/gsh/doctoral

7. Cornell -- Civil and Environmental Engineering. (n.d.). MS and PhD Candidate Assessment. Retrieved January 25, 2016, from http://www.cee.cornell.edu/academics/graduate/ms-phd-candidate-assessment.cfm

8. University of Virginia -- Career Development. (n.d.). Graduate Student Professional Skills and Competencies Checklist. Retrieved January 25, 2016, from https://gradcareer.virginia.edu/assessment-ofskills

9. Dreyfus, S. E., \& Dreyfus, H. L. (1980). A five-stage model of the mental activities involved in directed skill acquisition. University of California Berkeley Operations Research Center.

10. Peters, D. L., \& Daly, S. R. (2012). Why do professionals return to school for graduate degrees? Presented at the American Society of Engineering Education Annual Conference \& Exposition, San Antonio, TX.

11. Hofinger, R. J., \& Feldmann, L. J. (2001). The role of the adult student in the classroom. Presented at the American Society for Engineering Education Annual Conference \& Exposition, Albuquerque, NM.

12. MacFadgen, S. L. (2007). Mature students in the persistence puzzle: an exploration of the factors that contribute to adult learners' quality of life and retention in post-secondary education (Thesis). Faculty of Education - Simon Fraser University. Retrieved from http://summit.sfu.ca/item/8453

13. Prusak, Z. (1999). Learning environment in engineering technology with a high percentage of nontraditional Students. Presented at the American Society of Engineering Education Annual Conference \& Exposition, Charlotte, NC.

14. Mosyjowski, E. A., Daly, S.R., Peters D.L., \& Skerlos, S.J. (2013). Designing a Survey Instrument for a National Study of Direct-pathway and Returning Engineering Graduate Students. American Society of Engineering Education Annual Conference \& Exposition, Atlanta, GA.

15. Boyce, C., \& Neale, P. (2006). Conducting in-depth interviews: A guide for designing and conducting indepth interviews for evaluation input. Watertown, MA: Pathfinder International.

16. Brenner, M. E. (2006). Interviewing in educational research. Handbook of complementary methods in education research, 357-370.

17. Roulston, K., deMarrais, K., \& Lewis, J. B. (2003). Learning to interview in the social sciences. Qualitative Inquiry, 9(4), 643-668.

18. Bransford, J. (2000). How experts differ from novices. How People Learn: Brain, Mind, Experience, and School, 31-50.

19. Dreyfus, S. E. (2004). The Five-Stage Model of Adult Skill Acquisition. Bulletin of Science, Technology and Society, 24(3), 177-181.

20. Baxter Magolda, M. B. (2008). Three Elements of Self-Authorship. Journal of College Student Development, 49(4), 269-284. 